\title{
Quantitative Estimation of Interaction between Carbohydrates and Concanavalin A by Surface Plasmon Resonance Biosensor
}

\author{
Satoru Goto, ${ }^{*}$ Kyoko Masuda, Miyuki Miura, Keiko Kanazawa, Miki Sasaki, Miyuki Masui, \\ Maki Shiramizu, Hiroshi Terada, and Hiroshi Chuman \\ Faculty of Pharmaceutical Sciences, University of Tokushima, 1-78 Shomachi, Tokushima 770-8505, Japan. \\ Received August 8, 2001; accepted December 15, 2001
}

\begin{abstract}
We measured the affinity of more than 20 sugars with concanavalin A (ConA) by an optical biosensor (surface plasmon resonance sensor) using asialofetuin (ASF) as an immobilized binding partner of ConA. We determined kinetic parameters of the effects of sugars on the dissociation of ConA from ASF quantitatively, and the structural requirements of the functional groups of sugars for binding with ConA. We found that the affinity of ConA for sugars is dependent on its conformation induced by interaction with the binding partner. In addition, the results showed that optical biosensor system is well mimics the interaction of ConA with sugars in biomembrane.
\end{abstract}

Key words concanavalin A; asialo-fetuin; sugar interaction; optical biosensor

Concanavalin A (ConA) isolated from jack bean (Canavalia ensiformis) belongs to the legume lectin family. ${ }^{1)}$ Among the monosaccharides, methyl $\alpha$-D-mannopyranoside (Me $\alpha$-Man, see Chart) exhibits the most potent inhibitory effect on formation of the complex of ConA with dextran, ${ }^{2-4)}$ and the mannotriose 3,6-di- $O$-( $\alpha$-D-mannosyl)- $\alpha$-D-mannoside was found to be the most potent oligosaccharide, being more potent than Me $\alpha$-Man. ${ }^{5)}$

Shinohara et $a l^{6,7)}$ examined the binding of ConA with glycosidase-treated fetuins containing the branched mannotriose moieties, which were immobilized in the chip of an optical biosensor utilizing the surface plasmon resonance (SPR) technique. ${ }^{6-9)}$ Their results showed that the terminal mannotriose moiety of glucosaminidase-treated fetuin (aglucosaminofetuin) was most sensitive. Galactosidase-treated fetuin (agalactosylfetuin) and sialidase-treated fetuin (asialofetuin, ASF) having glycosylated non-terminal mannotriose moieties bound more weakly than aglucosaminofetuin to ConA. ${ }^{6}$

To understand the structural requirements of sugars for binding with ConA in biomembranes, we quantitatively analyzed the effects of various sugars on the dissociation of ConA from ASF using an optical biosensor system. As ASF does not interact with ConA tightly, ${ }^{7}$ the ASF-ConA system should be favorable for determination of the affinity of sugars for ConA. In fact, we found that our system is a good mode of interaction of sugars with ConA in biomembranes.

\section{Experimental}

Materials Human and bovine fresh blood was obtained from the Blood Center of the Japan Red Cross Society and the Meat Inspection Facility, Tokushima, respectively. ConA and ASF were purchased from Sigma. Other chemicals and reagents were of the highest grade commercially available.

Preparation of the ASF-Immobilized Dextran Matrix on the Biosensor Chip SPR measurements were performed with a BIACORE 1000 (Pharmacia Biosensor AB, Uppsala, Sweden). ${ }^{9)}$ We used a CM5 biosensor chip coated with carboxymethyldextran (CMD) matrix, and it was mounted in a flow-cell. The flow-cell was equilibrated with Hepes buffered saline (HBS) containing $90 \mathrm{~mm} \mathrm{NaCl}$ and $20 \mathrm{~mm}$ Hepes/ $\mathrm{NaOH}$ buffer (pH 7.0) at $25^{\circ} \mathrm{C}$, and CMD was activated with a mixture of $200 \mathrm{~mm} N$-ethyl- $N^{\prime}$-(diethylaminopropyl)-carbodiimide and $50 \mathrm{~mm} N$-hydroxysuccinimide. Into the activated flow-cell $30 \mathrm{mg} / \mathrm{ml} \mathrm{ASF}$ in $10 \mathrm{~mm}$ sodium acetate buffer ( $\mathrm{pH} 4.7$ ) was injected. Then, free activated carboxyl groups were masked by injection of $1 \mathrm{~m}$ ethanolamine at $\mathrm{pH} 8.5$. Each sample was injected into the ASF-im- mobilized flow-cell. The net amount of the conjugated ASF was calculated by subtracting its amount in the blank flow-cell from that of the ASF-immobilized flow-cell, in terms of SPR response. The immobilization of ASF to a CMD matrix in the flow-cell increased SPR by 2300 units, which may correspond to $2.3 \mathrm{ng} / \mathrm{mm}^{2} \mathrm{ASF}^{10)}$ The ASF-immobilized biosensor chip was stored at $4{ }^{\circ} \mathrm{C}$ in the chemically stable package.

Inhibition of Sugars on the Binding of Con A to Immobilized ASF A solution of $50 \mu \mathrm{g} / \mathrm{ml}$ ConA in HBS was injected into the ASF-immobilized flow-cell, and the flow-cell was washed with HBS for $15 \mathrm{~min}$. Then the test compound solution was applied for $15 \mathrm{~min}$. The interaction was monitored at $25^{\circ} \mathrm{C}$ as the change in the SPR response $(R)$. The same flow-cell was used repeatedly by washing it with $150 \mathrm{~mm} \mathrm{HCl}$.

The apparent dissociation rate constant $k_{\mathrm{d}}$ for the ConA-ASF complex was experimentally obtained by Eq. 1 , where $R$ and $R_{0}$ are the SPR responses at time $t$ and at the time of injection of the test compound $t_{0}$, respectively. ${ }^{6-8)}$

$$
\ln \frac{R_{0}}{R}=k_{\mathrm{a}}\left(t-t_{0}\right)
$$

The $\chi^{2}$-test was used to assess the validity of the obtained $k_{\mathrm{d}}$ value, using the operating program of the BIACORE 1000 system. ConA dissociated from the immobilized ASF at the surface of the sensor chip diffuses from the unstirred surface (stationary phase, $\mathrm{ConA}_{\mathrm{s}}$ ) to the mobile phase $\left(\mathrm{ConA}_{0}\right)$. These processes and the kinetics of dissociation of $\operatorname{ConA}_{\mathrm{s}}$ from ASF are shown by Eqs. 2 and 3, respectively.

$$
\begin{aligned}
& \mathrm{ASF}-\mathrm{ConA} \underset{k_{+}}{\stackrel{k_{-}}{\leftrightarrows}} \mathrm{ASF}+\mathrm{ConA}_{\mathrm{s}} \stackrel{k_{\mathrm{tr}}}{\longrightarrow} \mathrm{ConA}_{0} \\
& \frac{\mathrm{d} R}{\mathrm{~d} R}=k_{+}[\mathrm{ConA}]_{\mathrm{s}}\left(R_{0}-R\right)-k_{-} R
\end{aligned}
$$

Under steady-state conditions, $k_{\mathrm{d}}$ is given by Eq. 4 , where $k_{+}, k_{-}$, and $k_{\mathrm{tr}}$ represent intrinsic association and dissociation rate constants of ASF-ConA complex and mass-transport rate constant of ConA from the stationary phase to mobile phase, respectively. ${ }^{11,12)}$

$$
k_{\mathrm{d}}=\frac{k_{-}}{1+\frac{k_{+}\left(R_{0}-R\right)}{k_{\mathrm{tr}}}}
$$

The association constant $K_{\mathrm{i}}$ of the competitive inhibitor $\mathrm{S}$ with ConA is represented by Eq. 5 .

$$
K_{\mathrm{i}}=\frac{[\mathrm{ConA}-\mathrm{S}]}{[\operatorname{ConA}][\mathrm{S}]}
$$

Then, the relative concentration of free ConA $\alpha$ is shown by Eq. 6 . 


$$
\alpha=\frac{[\operatorname{Con} \mathrm{A}]}{[\operatorname{Con} \mathrm{A}]+[\operatorname{Con} \mathrm{A}-\mathrm{S}]}=\frac{1}{K_{\mathrm{i}}[\mathrm{S}]+1}
$$

When the binding of S to ConA proceeds immediately, Eq. 7 is obtained.

$$
\frac{\mathrm{d} R}{\mathrm{~d} t}=k_{+} \alpha[\operatorname{ConA}]_{\mathrm{s}}\left(R_{0}-R\right)-K_{-} R
$$

From Eqs. 3, 4 and 7, $k_{\mathrm{d}}$ is expressed by Eq. 8 .

$$
k_{\mathrm{d}}=\frac{k_{-}}{1+\frac{k_{+} \alpha\left(R_{0}-R\right)}{k_{\mathrm{tr}}}}
$$

Then, Eq. 9 is obtained.

$$
\frac{1}{k_{\mathrm{d}}}=\frac{1}{k_{-}}+\frac{k_{+}\left(R_{0}-R\right)}{k_{-} k_{\mathrm{tr}}} \frac{1}{K_{\mathrm{i}}[\mathrm{S}]+1}
$$

When $v=1 / k_{-}$and $\kappa=K_{\mathrm{i}} k_{\mathrm{tr}} / k_{+} /\left(R_{\mathrm{t}}-R\right)$, Eq. 10 is obtained from Eq. 9 under the conditions of $[\mathrm{S}] \gg 1 / K_{\mathrm{i}}$, showing that there is a linear relationship between $1 / k_{\mathrm{d}}$ and $1 /[\mathrm{S}]$.

$$
\frac{1}{k_{\mathrm{d}}}=v+\frac{v}{\kappa} \frac{1}{[\mathrm{~S}]}
$$

Values of $\kappa$ and $v$ are related to the association constant of S with ConA and the reciprocal of the intrinsic dissociation rate constant of ConA-ASF complex, respectively, and they are obtained by regression analysis computed with the software QSAR developed by us using Fortran $77 .{ }^{13-15}$ )

Erythrocyte Aggregation Test Human and bovine blood was washed three times with phosphate buffered saline (PBS) containing $150 \mathrm{~mm} \mathrm{NaCl}$ and $10 \mathrm{~mm}$ sodium phosphate buffer ( $\mathrm{pH}$ 7.4). The washed erythrocyte suspension in PBS was prepared at $2 \%(\mathrm{v} / \mathrm{v})$ of the packed cells. To the wells of a microtiter plate $50 \mu \mathrm{l}$ of $2 \%$ erythrocyte suspension and subsequently $100 \mu \mathrm{l}$ of test compound solution in PBS were added. The plate was kept at room temperature for $2 \mathrm{~h}$, and the degree of aggregation was scored.

\section{Results and Discussion}

Effects of Sugars on the ASF-ConA Complex Prior to conducting optical biosensor measurement, we confirmed the concentration dependence of ConA on its binding with ASF, which was covalently immobilized on the dextran bed in the optical biosensor chip. The immobilized ASF showed an SPR response of 2,300 units. ConA was added to the ASFimmobilized flow-cell, and injections of 50,100, 200, and $300 \mu \mathrm{g} / \mathrm{ml}$ of ConA induced the SPR responses of 2000, 4200, 8500, and 13000 units, respectively. In the blank flowcell without immobilized ASF, no increase of the SPR response was detected. Therefore, formation of the ASF-ConA complex in the flow-cell was nearly proportional to the concentration of the injected ConA. The following experiments were performed with $50 \mu \mathrm{g} / \mathrm{ml}$ ConA.

As shown in Fig. 1, the injection of ConA induced a hy-

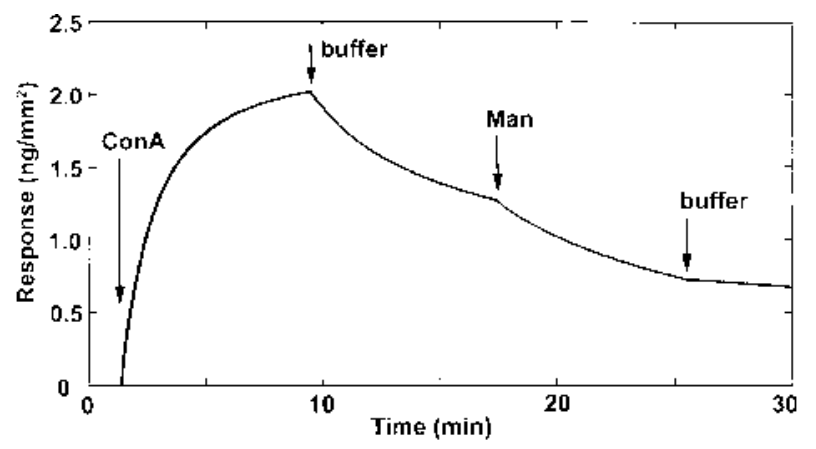

Fig. 1. Sensorgram Showing the Dissociation of ConA from the Immobilized ASF perbolic increase in the SPR response, showing that the association between ConA and the immobilized ASF was saturating. Subsequent injection of HBS buffer caused dissociation of ConA from the immobilized ASF. Injection of D-mannose (Man, see Chart) caused dissociation of ConA. Apparent rate constant $k_{\mathrm{d}}$ values for the dissociation of ConA induced by added sugars were measured and analyzed by Eq. 1 .

Increases in the $k_{\mathrm{d}}$ value induced by methyl $\alpha$-D-mannopyranoside (Me $\alpha$-Man, see Chart 1) are shown in Fig. 2. A similar curve was obtained for methyl $\alpha$-D-glucopyranoside (Me $\alpha$-Glc, see Chart 1). The observed dissociation of ConA from ASF was saturated at high concentrations of these sug-
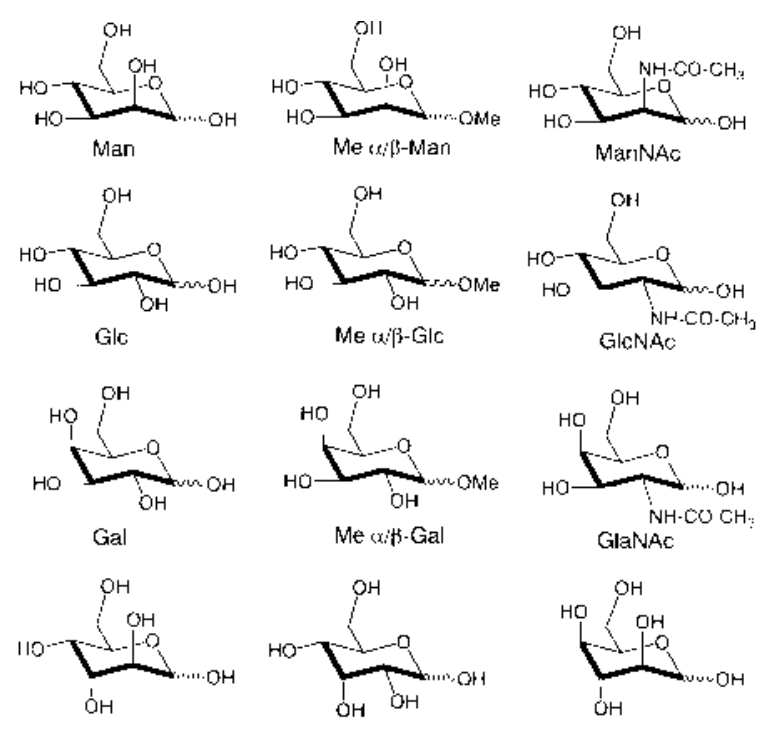

L-altrose

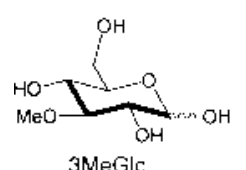

D-allose

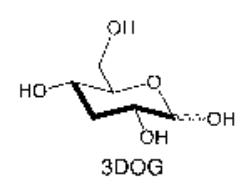

D-idose
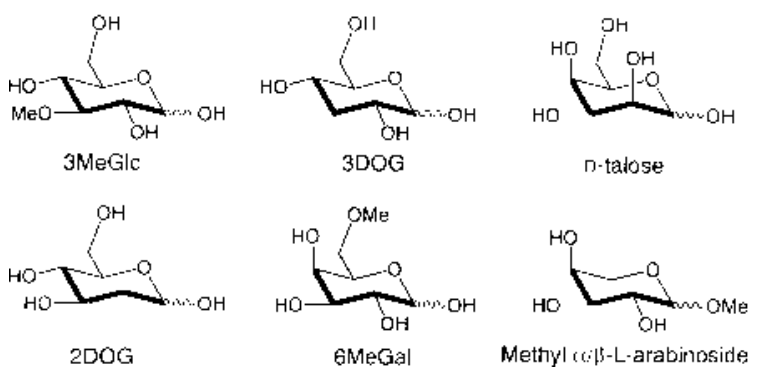

Chart 1. Chemical Structures of Sugars Examined in This Study

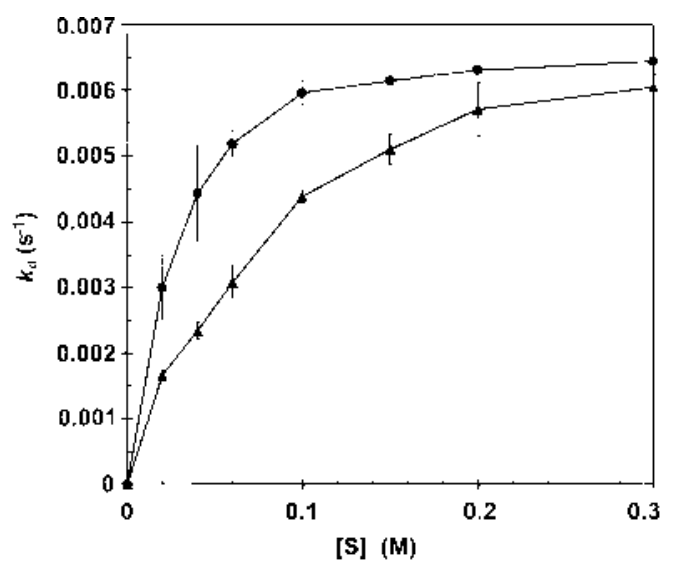

Fig. 2. Effects of Me $\alpha$-Man (O) and Me $\alpha$-Glc $(\boldsymbol{\Delta})$ on the Dissociation of the ASF-ConA Complex

The values are averages of 3 separate runs with \pm standard deviation. 


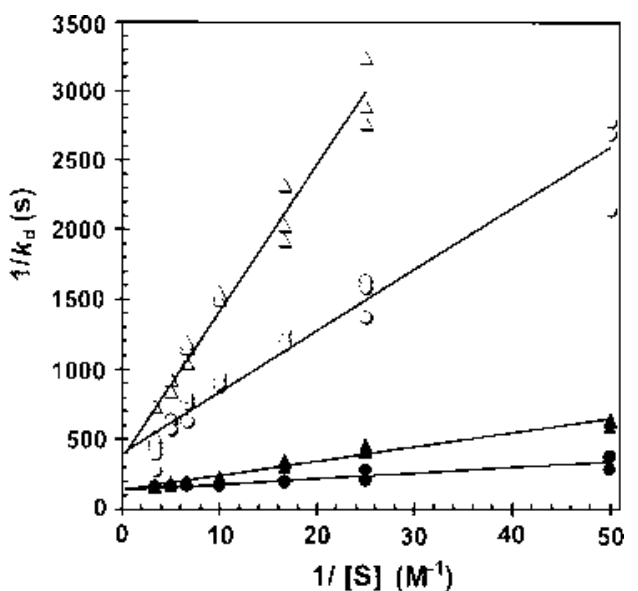

Fig. 3. Double Reciprocal Plots for Me $\alpha$-Man (O), Me $\alpha$-Man (A), Me $\beta$-Man (O), and $\operatorname{Me} \beta$-Glc $(\triangle)$

Table 1. Kinetic Parameters for Sugar Derivatives

\begin{tabular}{lcc}
\hline \hline Compound & $v(\mathrm{~s})$ & $\kappa\left(\mathrm{M}^{-1}\right)$ \\
\hline Me $\alpha$-Man & 135.0 & 32.9 \\
Me $\alpha$-Glc & 141.3 & 14.0 \\
Me $\beta$-Man & 396.1 & 9.02 \\
Me $\beta$-Glc & 359.4 & 3.39 \\
Man & 123.6 & 6.02 \\
Glc & 207.0 & 3.74 \\
2DOG & 187.4 & 4.79 \\
GlcNAc & 187.8 & 3.32 \\
ManNAc & 585.0 & 3.40 \\
\hline
\end{tabular}

ars. The double reciprocal plot between the measured $k_{\mathrm{d}}$ and the sugar concentration is shown in Fig. 3, and the affinity parameter $\kappa$ for each sugar was determined by Eq. 10. The results for Me $\alpha$-Man and Me $\alpha$-Glc are shown by Eqs. 11 and 12 , respectively,

$$
\begin{aligned}
& 1 / k_{\mathrm{d}}=135.0+4.097 /[\mathrm{Me} \alpha-\mathrm{Man}] \\
& \begin{array}{l}
( \pm 15.5) \quad( \pm 0.684) \\
(n=21, r=0.944, s=23.0
\end{array} \\
& \left(n=21, r=0.944, s=23.0, F_{1,19}=78.0\right) \\
& 1 / k_{\mathrm{d}}=141.3+10.06 /[\mathrm{Me} \alpha-\mathrm{Glc}] \\
& ( \pm 19.2) \quad( \pm 0.85) \\
& \left(n=21, r=0.985, s=28.6, F_{1,19}=305\right)
\end{aligned}
$$

where values in parentheses represent $95 \%$ confidence intervals, and $n, r, s$, and $F$ are the number of data points, correlation coefficient, standard deviation, and ratio of regression and residual variances, respectively.

From Eqs. 11 and 12, parameters related to association of the sugar to ConA $(\kappa)$ and reciprocal of the intrinsic dissociation of ConA-ASF complex $(v)$ were determined. Table 1 lists these values for $\operatorname{Me} \alpha$-Man and Me $\alpha$-Glc along with those of other sugars studied in this work described below. The value of $v$ for Me $\alpha$-Man well agreed with that for Me $\alpha$-Glc. The relative affinities $\kappa($ Me $\alpha$-Man) and $\kappa(\operatorname{Me} \alpha$ Glc) were determined to be $32.9 \mathrm{M}^{-1}$ and $14.0 \mathrm{M}^{-1}$, respectively, suggesting that the affinity of Me $\alpha$-Man for ConA is more than twice that of Me $\alpha$-Glc.

The selectivity of Me $\alpha$-Man to Me $\alpha$-Glc can be expressed by the affinity ratio of these sugars, i.e., $\kappa(\mathrm{Me} \alpha$ $\mathrm{Man}) / \kappa(\mathrm{Me} \alpha$-Glc $)=2.35$. The values of selectivity have been reported in terms of the ratio of $\mathrm{IC}_{50}$ values (the sugar concentration for induction of $50 \%$ dissociation of ConA from dextran or mannosyl-lipids) by various methods. Mann and his coworkers ${ }^{16)}$ reported the ratio 3.2 in their study on the effects of these sugars on the association of ConA with the immobilized mannosyl-lipids in an optical biosensor. Goldstein and his colleagues ${ }^{4,17)}$ obtained values of 5.7 and 4.5 from their inhibitory effects on dextran precipitation induced by ConA. These results suggest that the selectivity of Me $\alpha$-Man to ConA in its complex with the glucose moiety of dextran is greater than that in the complex of ConA with the mannose moiety of mannosyl-lipids. In contrast, the lower selectivity of Me $\alpha$-Man to Me $\alpha$-Glc of our present results could be due to the fact that the selectivity of ConA to Me $\alpha$-Man is decreased when ConA is bound to the mannotriose moiety of ASF.

Affinities of D-Mannose and D-Glucose to ConA Next, we examined the effects of methyl $\beta$-D-mannopyranoside (Me $\beta$-Man, see Chart 1) and methyl $\beta$-D-glucopyranoside (Me $\beta$-Glc, see Chart 1) on dissociation of ASF-ConA complex. The dependences of $1 / k_{\mathrm{d}}$ on $1 /[\mathrm{Me} \beta$-Man] and $1 /[\mathrm{Me}$ $\beta$-Glc] are shown in Fig. 3 and their relationships are expressed by Eqs. 13 and 14, respectively.

$$
\begin{gathered}
1 / k_{\mathrm{d}}=396.1+43.94 /[\mathrm{Me} \beta \text {-Man }] \\
( \pm 104.5) \quad( \pm 4.62) \\
\left(n=21, r=0.977, s=155, F_{1,19}=197\right) \\
1 / k_{\mathrm{d}}=359.4+105.6 /[\mathrm{Me} \beta \text {-Glc }] \\
( \pm 144.4) \quad( \pm 10.5) \\
\left(n=17, r=0.984, s=156, F_{1,15}=228\right)
\end{gathered}
$$

The values of $v$ and $\kappa$ thus determined are shown in Table 1. We obtained the value of 3.66 for the selectivity of Me $\alpha$ Man to Me $\beta$-Man (=k(Me $\alpha$-Man) $/ \kappa(\operatorname{Me} \beta$-Man)) from Eqs. 11 and 13, and 4.13 for that of Me $\alpha$-Glc to Me $\beta$-Glc $(=k(\mathrm{Me} \alpha$-Glc $) / \kappa(\mathrm{Me} \beta$-Glc $))$ from Eqs. 12 and 14 , showing that the affinity of $\alpha$-configuration of the 1-OMe is about 4times greater than the $\beta$-configuration for both methylatedMan and methylated-Glc. Although higher affinity of the $\alpha$ configuration than that of the $\beta$-configuration is known, we determined the selectivity quantitatively. In addition, $\kappa(\mathrm{Me}$ $\beta$-Man $) / \kappa($ Me $\beta$-Glc) was determined to be 2.65 from Eqs. 13 and 14.

As the values $\kappa(\mathrm{Me} \alpha-\mathrm{Man}) / \kappa(\mathrm{Me} \alpha$-Glc $)$ and $\kappa(\mathrm{Me}$ $\beta \mathrm{Man}) / \kappa(\mathrm{Me} \beta$-Glc) were very similar $(2.35$ and 2.65 , respectively)), the affinity of the configuration of the $2 \beta-\mathrm{OH}$ is more than twice that of $2 \alpha-\mathrm{OH}$, irrespective of the configuration of $1-\mathrm{OMe}(1 \alpha$-OMe for Me $\alpha$-Man and Me $\alpha$-Glc, and $1 \beta$-OMe for Me $\beta$-Man and Me $\beta$-Glc).

Next, we examined the effects of Man and D-glucose (Glc, see Chart 1) on the ASF-ConA complex. For this, we allowed these solutions to stand for $2 \mathrm{~d}$ to attain equilibrium, and measured SPR responses. We obtained Eqs. 15 and 16.

$$
\begin{gathered}
1 / k_{\mathrm{d}}=123.6+20.62 /[\mathrm{Man}] \\
( \pm 20.0) \quad( \pm 1.91) \\
\left(n=20, r=0.982, s=24.2, F_{1,18}=240\right) \\
1 / k_{\mathrm{d}}=207.0+55.33 /[\mathrm{Glc}] \\
( \pm 66.2) \quad( \pm 5.95) \\
\left(n=21, r=0.976, s=78.5, F_{1,19}=188\right)
\end{gathered}
$$

The affinity ratio $\kappa(\mathrm{Man}) / \kappa(\mathrm{Glc})$ was obtained as 1.60 , being 
smaller than the values of $\kappa(\operatorname{Me} \alpha-\operatorname{Man}) / \kappa(\mathrm{Me} \alpha$-Glc $)$ $(=2.35)$ and $\kappa(\operatorname{Me} \beta$-Man $) / \kappa(\operatorname{Me} \beta$-Glc) $(=2.65)$. The equilibrium concentrations of $\alpha$ - and $\beta$-mannopyranoses in water are known to be $68 \%$ and $32 \%$, respectively, and those of $\alpha$ and $\beta$-glucopyranoses are $36 \%$ and $64 \%{ }^{18)}$ From the ${ }^{1} \mathrm{H}$ NMR spectra at a concentration of $10 \mathrm{~mm}$ of the anomeric mixture of these sugars in $\mathrm{D}_{2} \mathrm{O}$, we obtained nearly the same values (data not shown). Using these values, we obtained Eqs. 17 and 18 for the anomeric mixtures of Man and Glc, respectively.

$$
\begin{aligned}
& \kappa(\mathrm{Man})=0.68 \kappa(\alpha \text {-Man })+0.32 \kappa(\beta \text {-Man }) \\
& \kappa(\mathrm{Glc})=0.36 \kappa(\alpha \text {-Glc })+0.64 \kappa(\beta \text {-Glc })
\end{aligned}
$$

As the affinities of $1 \alpha$-OMe and $1 \beta$-OMe were both independent of the configuration of the $2-\mathrm{OH}$, it can be assumed that the same relationship is observed for sugars having the $1-\mathrm{OH}$ groups such as $\alpha$-Man, $\beta$-Man, $\alpha$-Glc and $\beta$-Glc. Hence the following relations are derived.

$$
\begin{aligned}
& \kappa(\alpha \text {-Man }) / \kappa(\beta \text {-Man })=k(\text { Me } \alpha \text { Man }) / \kappa(\text { Me } \beta \text {-Man })=3.66 \\
& \kappa(\alpha \text {-Glc }) / \kappa(\beta \text {-Glc })=k(\text { Me } \alpha \text { Glc }) / \kappa(\text { Me } \beta \text {-Glc })=4.13
\end{aligned}
$$

From Eqs. $17-20$, we obtained $\kappa(\alpha$-Man $)=7.84, \kappa(\beta$ Man $)=2.14, \kappa(\alpha$-Glc $)=7.26$ and $\kappa(\beta$-Glc $)=1.76$.

From these values, the selectivities $\kappa(\alpha$-Man $) / \kappa(\alpha$-Glc $)$ and $\kappa(\beta$-Man $) / \kappa(\beta$-Glc $)$ were deduced as 1.08 and 1.22 , respectively, being quite different from $\kappa(\operatorname{Me} \alpha$-Man $) / \kappa($ Me $\alpha$ $\mathrm{Glc})=2.35$ and $\kappa(\mathrm{Me} \beta$-Man $) / \kappa(\mathrm{Me} \beta$-Glc $)=2.65$. It is noteworthy that the selectivity of the $2-\mathrm{OH}$ changed greatly for the sugars with 1-OMe and 1-OH, consistent with the results that the $2 \alpha-\mathrm{OH}$ group of Glc is not directly associated with the binding to ConA, whereas the $2 \beta-\mathrm{OH}$ is associated with the binding. These results were consistent with the crystallographic structures of ConA-Me $\alpha$-Man and ConA-Me $\alpha$-Glc complexes. $^{19,20)}$

Affinities of Sugars and Related Compounds to ConA The effects of the other hexoses: D-galactose (Gal), D-allose, D-altrose, D-idose and D-talose on the ASF-ConA complex were also studied with the optical biosensor. Chemical structures of these sugars are shown in the Chart 1. These sugars did not affect the ConA binding to ASF even at $300 \mathrm{~mm}$. The effect of D-gulose could not be examined because of its low solubility. As these hexoses do not contain either the $3 \beta-\mathrm{OH}$ or $4 \alpha-\mathrm{OH}$, these $\mathrm{OH}$ groups are suggested to be essential for binding with ConA.

Besides these hexoses, the effects of 14 sugars and their related compounds (chemical structures, see Chart 1) on the ASF-ConA complex were further tested up to $300 \mathrm{~mm}$. We found that 2-deoxy-D-glucose (2DOG), $N$-acetyl-D-glucosamine (GlcNAc) and $N$-acetyl-D-mannosamine (ManNAc) enhanced the dissociation of the ASF-ConA complex, but 3-deoxy-D-glucose (3DOG), 3- $O$-methyl-D-glucose (3MeGlc), methyl $\alpha$-D-galactopyranoside (Me $\alpha$-Gal), methyl $\beta$-D-galactopyranoside (Me $\beta$-Gal), 6- $O$-methyl-D-galactose, $N$-acethylD-galactose (GalNAc), methyl $\beta$-D-arabinopyranoside, methyl $\beta$-L-arabinopyranoside, brefeldin A and cytochalasin $\mathrm{B}^{15)}$ had no significant effect. Lack of the effects of $3 \mathrm{DOG}$ and $3 \mathrm{MeGlc}$ indicated that the $3 \beta-\mathrm{OH}$ group of Man and Glc acts as a proton donor for hydrogen bonding with ConA.

To clarify the role of the functional groups at the C2-position, we next examined the dose-dependent effects of $2 \mathrm{DOG}$ and GlcNAc, which do not have the 2-OH group, and ob- tained the relations shown in Eqs. 21 and 22, respectively.

$$
\begin{gathered}
1 / k_{\mathrm{d}}=187.4+39.16 /[2 \mathrm{DOG}] \\
( \pm 53.9) \quad( \pm 5.00) \\
\left(n=15, r=0.978, s=53.5, F_{1,13}=141\right) \\
1 / k_{\mathrm{d}}=187.8+56.67 /[\mathrm{GlcNAc}] \\
( \pm 88.0) \quad( \pm 7.72) \\
\left(n=21, r=0.962, s=101, F_{1,19}=117\right)
\end{gathered}
$$

There is a relation of $\kappa(\operatorname{Man})(=6.02)>k(2 \mathrm{DOG})(=$ $4.79)>k(\mathrm{Glc})(=3.74) \approx \kappa(\mathrm{GlcNAc})(=3.32)$. Therefore, it is suggested that the $2 \beta-\mathrm{OH}$ (Man) is associated with the binding to ConA, and the $2 \alpha$-acetamido (GlcNAc) and $2 \alpha-\mathrm{OH}$ (Glc) groups decrease the binding. As the values of $\kappa(\alpha$ Man $) / \kappa(\alpha$-Glc $)$ and $\kappa(\beta$-Man $) / \kappa(\beta$-Glc $)$ were close to 1 , configuration of the 2-OH group was not associated with the binding with ConA. Therefore, the intermediate value of $\kappa(2 \mathrm{DOG})$ could be due to the same populations of the $1 \alpha$ $\mathrm{OH}$ and $1 \beta-\mathrm{OH}$ anomers.

We also obtained Eq. 23 for ManNAc.

$$
\begin{gathered}
1 / k_{\mathrm{d}}=\quad 585.0+\quad 172.0 /[\mathrm{ManNAc}] \\
( \pm 471.0) \quad( \pm 52.2) \\
\left(n=10, r=0.935, s=293, F_{1,8}=27.8\right)
\end{gathered}
$$

The value of $\kappa$ (ManNAc) was determined as 3.40 , being much less than $\kappa$ (Man). Therefore, substitution of $2 \beta-\mathrm{OH}$ of Man by the acetamido-group decreased the binding affinity, possibly due to the steric hindrance of the acetamido-group. The $v$ value of ManNAc was 585, much greater than those of other sugars in the range of $120-400$ (see, Table 1). This could be because $K_{\mathrm{i}}$ of ManNAc was not great enough to satisfy the requirement of the experimental conditions $[\mathrm{S}] \gg$ $1 / K_{\mathrm{i}}$ in Eq. 9. Therefore, it is possible that the value of $\kappa$ (ManNAc) has not been determined exactly by Eq. 23.

Effect of Sugars on the Erythrocyte Aggregation Induced by ConA To know the roles of the functional groups at the C2-position of sugars in interaction with ConA by a method other than SPR sensor, we examined the effects of the sugars Man, Glc, GlcNAc and ManNAc on the aggregation of erythrocytes induced by ConA, which interacts tightly with the mannotriose moieties of the erythrocyte membrane surface. We found that the sugar chains of the band-3 protein in bovine erythrocytes were not highly sensitive to lectins, but those of human erythrocytes are sensitive (data not shown), possibly because the population of mannotriose moiety on human erythrocyte membrane is higher that on bovine erythrocyte membrane.

The addition of $30 \mu \mathrm{g} / \mathrm{ml}$ ConA to human erythrocytes at $0.7 \%(\mathrm{v} / \mathrm{v})$ suspended in saline induced cell aggregation. The effects of sugars on the ConA-induced aggregation were assayed in 96-well microtiter plates. Man, Glc, GlcNAc, and ManNAc inhibited the ConA-induced cell aggregation. The minimum inhibitory concentration (MIC) was estimated as $0.3 \mathrm{~mm}$ for Man, $1.5 \mathrm{~mm}$ for Glc, $5.0 \mathrm{~mm}$ for GlcNAc, and $20 \mathrm{~mm}$ for ManNAc. It is noteworthy that ManNAc showed the inhibitory effect, although its effect was not potent.

We also observed the aggregation of bovine erythrocytes $(0.7 \%(\mathrm{v} / \mathrm{v}))$ on addition of the higher concentration of $300 \mathrm{mg} / \mathrm{ml}$ of ConA, due to lower ConA affinity to bovine erythrocytes than to human erythrocytes. The MIC values were $24 \mathrm{~mm}$ for Man, $0.1 \mathrm{M}$ for Glc, $0.15 \mathrm{M}$ for GlcNAc, and 
$0.3 \mathrm{M}$ for ManNAc, being much higher than those in human erythrocytes.

Using dextran-ConA precipitation test, Poretz and Goldstein $^{4)}$ reported the $\mathrm{IC}_{50}$ values of $2.0 \mathrm{~mm}$ for Man, $11.3 \mathrm{~mm}$ for Glc, and approximately $13 \mathrm{~mm}$ for GlcNAc. ManNAc showed only a partial effect. These results suggest that the affinity of sugars to ConA is dependent on the binding partner of ConA, i.e., mannotrioses in erythrocyte membrane and the Glc moiety of dextran in the assay system of Poretz and Goldstein. ${ }^{4}$ Therefore, our results showed that the sugar recognition of ConA is more sensitive in the conformation of ConA induced by binding with mannotrioses than that induced by dextran. As ASF contains mannotriose moiety, our present system well mimics biomembranes.

In conclusion, we found that the affinity of sugars for ConA is dependent on the binding partner of ConA. The conformation of ConA possibly changes on binding with the binding partner, and this conformational change then affects the binding to sugars. In the present ConA-ASF system, the binding affinity of $\alpha$-Glc was closer to that of $\alpha$-Man, and introduction of the non-methylated group to the 1-OH of these sugars considerably decreased the sugar selectivity to ConA.

Acknowledgements The authors are grateful to Prof. Takenori Kusumi, Dr. Mohamad Radwan Almofti (Faculty of Pharmaceutical Sciences, University of Tokushima), and Dr. Tatsuo Munakata (Tohwa Institute for Orient Studies, Tohwa University) for valuable discussion. This research was supported by Grants-in-aid for Scientific Research 13771355 and 11771470 (to S.G.), and 11672215 (to H.C.) from the Ministry of Education, Culture, Sports, Science and Technology (MEXT) of Japan.

\section{References}

1) Sumner J. B., Howell S. F., J. Biol. Chem., 115, 583-588 (1936).

2) Goldstein I. J., Hollerman C. E., Merrick J. M., Biochim. Biophys. Acta, 97, 68-76 (1965).

3) Goldstein I. J., Hollerman C. E., Smith E. E., Biochemistry, 4, 876883 (1965).

4) Poretz R. D., Goldstein I. J., Biochemistry, 9, 2890-2896 (1970).

5) Debray N., Decout D., Strecker G., Spik G., Montreuil J., Eur. J. Biochem., 117, 41-55 (1981).

6) Shinohara Y., Sota H., Kim F., Shimizu M., Gotoh M., Tosu M., Hasegawa Y., J. Biochem. (Tokyo), 117, 1076-1082 (1995).

7) Okazaki I., Hasegawa Y., Shinohara Y., Kamasaki T., Bhikhabhai R., J. Mol. Recognit., 8, 95-99 (1995).

8) Chaiken I., Rose S., Karlsson R., Anal. Biochem., 201, 197-210 (1992).

9) Imata H., Kubota K., Hattori K., Aoyagi M., Jindoh C., Bioorg. Med. Chem. Lett., 7, 109-112 (1997).

10) Nagata K., Handa H., "Protocols for Real Time Analyses of Biological Interactions with BIACORE," Springer-Verlag Tokyo, 1998, Tokyo.

11) Glaser R. W., Anal. Biochem., 213, 152-161 (1993).

12) Schuck P., Minton A. P., Anal. Biochem., 240, 262-272 (1996).

13) Terada H., Goto S., Yamamoto K., Takeuchi I., Hamada Y., Miyake K., Biochim. Biophys. Acta, 936, 504-512 (1988).

14) Goto S., Guo Z. R., Futatsuishi Y., Hori H., Taira Z., Terada H., J. Med. Chem., 35, 2440-2445 (1992).

15) Goto S., Chen K. X., Oda S., Hori H., Terada H., J. Am. Chem. Soc., 120, 2457-2463 (1998).

16) Mann D. A., Kanai M., Maly D. J., Kiessling L. L., J. Am. Chem. Soc., 120, 10575-10582 (1998).

17) Scott J. K., Loganathan D., Easley R. B., Gong X., Goldstein I. J., Proc. Natl. Acad. Sci. U.S.A., 89, 5398-5402 (1992).

18) Reeves R. E., J. Am. Chem. Soc., 72, 1499-1506 (1950).

19) Naismith J. H., Emmerich C., Habash J., Harrop S. J., Helliwell J. R., Hunter W. N., Raftery J., Kalb (Gilboa) A. J., Yariv J., Acta Crystallogr. Sect., D50, 847-858 (1994).

20) Harrop S. J., Heliwell J. R., Wan T. C. M., Kalb (Gilboa) A. J., Tong L., Yariv J., Acta Crystallogr. Sect., D52, 143-155 (1996). 